\title{
Prueba computarizada Memonum: efecto de intervalos y distractores sobre la memoria de trabajo en mujeres mayores de 50 años ${ }^{*}$
}

\author{
Memonum Computerized Test: The Effects of Intervals and \\ Distractors on Working Memory in Women Over 50 Years of Age
}

Recibido: febrero 2 de 2010 | Revisado: mayo 24 de 2010 | Aceptado: agosto 6 de 2010

\author{
Edward Leonel Prada Sarmiento** \\ Universidad Pontificia Bolivariana, Bucaramanga, \\ Colombia \\ Gina Elizabeth Pineda Garzón **** \\ Manuel Alejandro Mejía Orduz \\ UIS-UPB (NYC), Bucaramanga, Colombia \\ Carlos Arturo Conde Cotes \\ Universidad Industrial de Santander, Colombia
}

Para citar este artículo. Prada Sarmiento, E.L., Pineda Garzón, G.E., Mejía Orduz, M.A. \& Conde Cotes, C.A. (2010). Prueba computarizada Memonum: efecto de intervalos y distractores sobre la memoria de trabajo en mujeres mayores de 50 años. Universitas Psychologica, 9 (3), 893-906.

* Artículo de investigación en neuropsicología.

** Facultad de Psicología, Laboratorio de Neurociencias y Comportamiento, Universidad Pontificia Bolivariana, Bucaramanga, Colombia, A.A. 2932. Correo electrónico: edward.prada@upbbga.edu.co

**** Grupo de Neurociencias y Comportamiento UISUPB (NYC), Bucaramanga, Colombia. Correo electrónico: psicomano@gmail.com

****** Departamento de Ciencias Básicas, Facultad de Salud, Laboratorio de Neurociencias y Comportamiento, Universidad Industrial de Santander, A.A.

RES UMEN

Mediante la prueba computarizada Memonum se evaluaron los efectos de dos intervalos de exposición de dígitos (1 y 8 segundos), y la presentación de un distractor sobre el desempeño de la memoria de trabajo en 80 mujeres mayores de 50 años. Se evidenció un efecto significativo del intervalo sobre las variables: número de aciertos, aciertos acumulados, y empleo de estrategias adicionales, demostrando un mejor desempeño mnemónico en participantes que presentaron la prueba en el intervalo de 8 segundos y sugiriendo que un tiempo amplio de exposición potencia la capacidad de almacenamiento de la memoria en adultos mayores. La presencia del distractor tuvo un efecto significativo sobre el número de aciertos acumulados, considerándose como un estimulo interferente capaz de afectar la memoria de trabajo en adultos mayores.

Palabras clave autores

Memoria de trabajo, prueba computarizada Memonum, intervalos de exposición, distractor.
A B S T R A C T
By means of the Memonum computerized test the effects of two exhibition intervals of digits ( 1 and 8 seconds), and the presentation of a distracter were evaluated on the working memory performance in 80 women older than 50 years. Significant effect of the exhibition interval on the number of successes, total successes and use of additional strategies it was revealed, demonstrating better mnemonic performance of participants that presen- ted the test in the interval of 8 seconds and suggesting that a wide time of contact with the information power the memory's storage capacity in older adults. The distracter presence had a significant effect on total successes variable, considered as interfere stimulus able to affect the working memory in older adults.
Keywords authors
Working Memory, Memonum Computerized Test, Exhibition Intervals, Distracter. 


\section{Introducción}

Las neurociencias prestan gran interés a la memoria de trabajo u operativa, caracterizada por su corta duración y uso habitual, que posibilita la manipulación flexible de la información, aunque en condiciones limitadas de tiempo y capacidad de elementos para procesar (Repovs \& Baddeley, 2006). Resulta necesaria en el recuerdo episódico y semántico, la toma de decisión, la comprensión del lenguaje, el cálculo mental, la ubicación espacial y el aprendizaje (Burin \& Duarte, 2005; Rodríguez, Fajardo \& Mata, 2006).

Dicho modelo cognitivo está conformado básicamente por tres componentes. El ejecutivo central, consiste en un controlador voluntario relacionado con la experiencia consciente, modula la atención, coordina estrategias mentales y manipula información bajo los requerimientos de una tarea (Etchepareborda \& Abad-Mas, 2005); los dos restantes, catalogados como sistemas subordinados son: el bucle fonológico, responsable de información basada en el lenguaje, de tareas lingüísticas, como la comprensión lectora, la escritura, la conversación; y, la agenda visoespacial, involucrada en la creación y manipulación de información visual, la aptitud espacial, la ubicación, el desplazamiento y en tareas que suponen memoria espacial (Baddeley, 1999; Gathercole, 1994).

En este repertorio funcional de la memoria de trabajo, se hallan implícitos procesos adicionales como la atención, que permite seleccionar información sensorial relevante, ignorando a su vez estímulos no relevantes como parte de un comportamiento dirigido hacia un objetivo preciso (Estévez, García \& Junqué, 1997; Pousada, 1998).

La memoria, como otros procesos cognoscitivos, permanece sujeta a cambios del desarrollo; Ardila y Roselli (1994) señalan que, a partir de los 50 años de edad, ocurre una clara disminución en la memoria reciente, la adquisición y el recuerdo de nueva información. Si bien, desde la mediana edad, la queja más frecuente acerca del funcionamiento cognitivo, está asociada con la pérdida de memoria en la vida diaria (Uribe, Valderrama
\& Molina, 2007), Crook et al. (1986) advierten que, a pesar de una disminución del rendimiento en test de memoria, se mantienen preservadas las funciones generales, sin presentar demencia o un trastorno mental específico.

Diversas son las posturas en torno a la idea de un deterioro de la memoria de trabajo en adultos mayores. Gazzaley, Sheridan, Cooney y D'Esposito (2007) plantean una disminución en la capacidad para retener información, mientras se realizan operaciones de procesamiento. Por su parte, Pousada (1998) propone la pérdida de memoria asociada a la edad, basada en un déficit de los mecanismos atencionales ejercidos por el sistema ejecutivo, provocando distractibilidad, mayor frecuencia de olvidos y respuestas inapropiadas. Autores como Román y Sánchez (1998) y Salthouse (1996) formulan que este deterioro, se caracteriza por mayor demanda en el tiempo de procesamiento, manipulación y recuperación de la información, mientras que el volumen de almacenamiento podría no diferir significativamente del de un adulto joven.

Para el abordaje de cambios cognitivos, se sugiere la creación de instrumentos computarizados que posibiliten homogenizar y controlar la presentación de los estímulos (tamaño, color, posición, intervalos de exposición), estableciendo condiciones que no permiten los formatos tradicionales como el empleo de distractores atencionales, e incluso la medición de variables adicionales al número de aciertos, como son los tiempos de respuesta, los cuales se relacionan con la velocidad del procesamiento cognitivo (Rodríguez et al., 2006).

El estudio de la memoria de trabajo no ha sido ajeno al empleo de herramientas computacionales. Emery, Myerson y Hale, (2007) emplearon diferentes intervalos de presentación de secuencias dígito-letra (750, 1750 o 2750 milisegundos), investigando en jóvenes y adultos mayores mecanismos de procesamiento de la información. Fischer (2001) administrando una versión computarizada de la Tarea de Cubos de Corsi en tres intervalos de presentación (1, 3 y 9 segundos), demostró un mejoramiento en la capacidad de almacenamiento en función de un mayor tiempo de exposición. De 
hecho, el tiempo de exposición de la información se considera un recurso potenciador de la capacidad mnemónica de adultos mayores, en programas de entrenamiento cognitivo asistidos por computadora (Günther, Schäfer, Holzner \& Kemmler, 2003; Lynch, 2002). Por su parte, Rodríguez et al. (2006), describen una versión computarizada de la subescala de dígitos WAIS, que evalúa la memoria a corto plazo, exponiendo series numéricas en orden progresivo o regresivo, e involucra el uso de elementos distractores, en el abordaje de interferencias sobre el proceso atencional.

Ante este panorama, el Grupo de Neurociencias y Comportamiento UIS-UPB, diseñó la prueba de retención numérica Memonum, instrumento computarizado que permite simultáneamente manipular los intervalos de exposición, incluir cambios de colores como estímulos distractores, y registrar variables relacionadas con aciertos y tiempos de respuesta. Albarracín, Dallos y Conde (2008) implementaron dicha prueba en estudiantes universitarios, encontrando un aumento del desempeño mnemónico en función del incremento de los intervalos de exposición (1, 8 y 16 segundos.), y un efecto de perturbación atencional sobre los tiempos de respuesta generado por el distractor; perfilando al Memonum como herramienta útil en la evaluación de la memoria de trabajo.

El presente estudio se interesó por abordar la memoria de trabajo en personas mayores de 50 años, de acuerdo con los cambios cognitivos enunciados: mínima disminución en el volumen mnemónico (Ardila \& Roselli, 1994; Gazzaley et al., 2007), lentificación en la respuesta consecuencia de una afectación en el procesamiento cognitivo (Salthouse, 1996), y dificultades en la selección atencional o en la inhibición de estímulos irrelevantes (Burin \& Duarte, 2005; Pousada, 1998). De modo que el objetivo de esta investigación fue evaluar el efecto de dos intervalos de exposición de dígitos (1 y 8 segundos), y la presentación de un distractor sobre el desempeño mnemónico en la Prueba Memonum).

\section{Método}

\section{Diseño}

Investigación de tipo experimental con distribución aleatoria por bloques, en el cual se implementó un diseño factorial $2 \times 2$, siendo el primer factor el intervalo de exposición de dígitos en la Prueba Memonum (1 y 8 segundos) y el segundo factor el tipo de presentación de las secuencias numéricas (presencia y ausencia de distractor).

\section{Participantes}

Participaron de manera voluntaria, consentida e informada 80 personas de género femenino, pertenecientes a grupos de la tercera edad de Floridablanca, con edades entre 50 y 72 años, y escolaridad comprendida entre 3 y 13 años de estudio. Cada evaluado fue asignado aleatoriamente a uno de los grupos de investigación: intervalo de $1 \mathrm{~s}$ presencia de distractor (1PD), intervalo de $1 \mathrm{~s}$ ausencia de distractor (1AD), intervalo de 8 $\mathrm{s}$ presencia de distractor (8PD) e intervalo de $8 \mathrm{~s}$ ausencia de distractor (8AD); manteniendo un número equivalente $(n=20)$.

La selección de la muestra fue intencional, utilizando como criterios de inclusión una edad igual o mayor a 50 años, y una escolaridad de 3 o más años aprobados. Los criterios de exclusión involucraron: reporte de dificultades auditivas o visuales no corregidas; alteraciones motrices; antecedentes de patologías neurológicas, enfermedades psiquiátricas, problemas médicos significativos, dependencia de agentes tóxicos o uso de psicofármacos. Así mismo, fueron excluidos quienes presentaron fallas en el reconocimiento de dígitos; posible alteración cognitiva valorada por el Examen Mental Abreviado (EMA), o sintomatología depresiva clínicamente relevante detectada por la Escala de Depresión del Centro de Estudios Epidemiológicos (CES-D). 


\section{Materiales y Procedimiento}

Los participantes fueron evaluados individualmente en dos sesiones (tamizaje y experimental), desarrolladas en días consecutivos y en horarios iguales o similares.

Sesión de tamizaje (40 minutos). Firmado el consentimiento informado, se implementó el siguiente protocolo: Ficha de Ingreso, EMA y CESD. Los instrumentos fueron administrados por el evaluador, contrastando los datos obtenidos con los criterios de exclusión.

Ficha de Ingreso. Formato diseñado para examinar la probable presencia de dificultades sensoriales, fallas en el reconocimiento visual o auditivo de dígitos, antecedentes neurológicos, psiquiátricos, médicos, toxicológicos y psicofarmacológicos.

EMA. Versión traducida al español del Test Mini-Mental de Folstein, Folstein y McHugh (1975), adaptada al contexto colombiano para la valoración del estado cognoscitivo global (Roselli et al., 2000). Consta de 30 ítems que evalúan: orientación, retención, atención, cálculo, evocación, lenguaje y praxia; para la puntuación de cada ítem se asignó el valor de 0 si la ejecución era incorrecta o 1 si era correcta. De acuerdo con el desempeño en el EMA, fueron excluidos los participantes que presentaron posible alteración cognoscitiva, tomando como parámetros una calificación igual o menor a 21, 24 y 26 puntos, para personas con 3 a 5, 6 a 12 , y más de 12 años de estudio, respectivamente (Pradilla et al., 2002).

CES-D. Traducción realizada por Orozco et al. (2004) de la escala CES-D (Radloff, 1977). Esta prueba evalúa sintomatología depresiva mediante 20 ítems con cuatro opciones de respuesta que cuantifican la frecuencia de experimentación de los fenómenos descritos por los reactivos durante la última semana, incluyendo el día actual; 16 de los ítems son de contenido negativo (valor de $\mathrm{Oa}$ $3)$; mientras los restantes presentan una connotación positiva (valor 3 a 0). Se utilizó como criterio de exclusión de participantes con sintomatología depresiva clínicamente relevante, una calificación igual o mayor a 16 puntos (Orozco et al., 2004; Radloff, 1977).
Sesión experimental (20 minutos). Los participantes fueron asignados aleatoriamente a uno de los grupos de investigación (1PD, 1AD, 8PD y $8 \mathrm{AD})$ y a un orden de administración de los instrumentos, según el cual la mitad de los evaluados de cada grupo presentaron el Test de Cancelación de la "A" antes o después de la prueba Memonum y del Formato de Autoinforme; efectuando así un método de balanceo.

Prueba Memonum. Prueba computarizada diseñada por el Grupo de Neurociencias y Comportamiento UIS-UPB, para la evaluación de memoria de trabajo (Albarracín et al., 2008).

Consistió en la presentación de una secuencia de números aleatorios de una cifra ( 0 a 9), los cuales fueron expuestos uno a uno, en el centro de la pantalla del computador, durante un intervalo de $1 \mathrm{u} 8$ segundos. Concluido el intervalo, y sin límite de tiempo, el participante debía digitar en el orden exacto la serie observada hasta el número actual. Si la respuesta era correcta, aparecía un nuevo dígito y, si era incorrecta, el programa se detenía. Por ejemplo: en el primer ciclo, el programa muestra el número 5; a continuación, el evaluado debe digitar "5". En el siguiente ciclo, se presenta el número 9; por tanto, el evaluado debe digitar "5" y "9"; y así sucesivamente hasta cometer un error.

Adicionalmente, el software permitió seleccionar el tipo de presentación de la secuencia numérica; en ausencia de distractor el fondo de la pantalla era de color negro, y en presencia de distractor se incluían cambios en los colores del fondo a partir del segundo dígito, este componente pretendió evaluar la capacidad de la herramienta para detectar interferencias atencionales. Los dígitos fueron siempre de color blanco en estilo Lucida Console, tamaño 36.

Para la aplicación del Prueba Memonum, se empleó un computador portátil procesador 1.6 $\mathrm{GHz}$ con pantalla LCD 17 pulgadas, y un teclado numérico con dispositivo USB. Cada participante se ubicó frente al computador a una distancia aproximada de 40 centímetros.

Inicialmente, se desarrolló una rutina de entrenamiento orientada a ofrecer instrucciones y conocimiento sobre la Prueba. Los evaluados 
realizaron tres ensayos en ausencia de distractor, utilizando el intervalo (1 u 8 segundos) según el grupo de investigación al que pertenecieran. En esta rutina, los datos derivados del desempeño no fueron almacenados por el programa.

Posteriormente, se ejecutó una rutina de evaluación. Los participantes efectuaron un ensayo con el mismo intervalo de exposición empleado en el entrenamiento, utilizando el tipo de presentación (presencia o ausencia de distractor) estipulado por el grupo de investigación del cual formaran parte. El programa registró las características técnicas del ensayo, el número de aciertos y los tiempos de respuesta en la digitación de cada número.

Para evitar que el nivel de dificultad de las series numéricas se constituyera en una variable extraña, se implementaron las mismas secuencias de dígitos y en el mismo orden, para todos los evaluados; éstas se seleccionaron de los 100 archivos que dispone el programa.

El software que funciona en sistema DOS y lenguaje "GWBASIC”, fue manipulado por el evaluador; los participantes sólo operaron el teclado numérico para digitar las series observadas.

Formato de Autoinforme. Dicho formato ponderó el valor que el participante asignaba a tres aspectos de su desempeño en la Prueba Memonum: estrategias empleadas, nivel de distracción y nivel de dificultad (Albarracín et al., 2008). Fueron examinadas las siguientes estrategias para el mantenimiento de la serie numérica: Secuencias de Digitación (ubicación de los números y la trayectoria de digitación en el teclado numérico), Repetición Mental (repaso de la secuencia de dígitos), Visualización Mental (creación de imágenes), y Otras estrategias (referidas por el evaluado). Para la administración del instrumento se solicitó al participante calificar los aspectos en escalas de 0 a 10, siendo 0 el mínimo valor y 10 el máximo.

Test de Cancelación de la "A". Fue concebido como una medida de vigilancia y registro visual que valora la atención selectiva y sostenida (Ardila, Rosselli \& Puente, 1994; Estévez et al., 1997; Lezak, 1995). El participante debía cancelar todas las letras "A" que encontrara en una planilla con 160 letras. Fueron calificados los aciertos (máximo
16). Adicionalmente, se registró el tiempo total de ejecución. Este instrumento se empleó para obtener un registro de los procesos atencionales, que posibilitara determinar su relación con el desempeño en la Prueba Memonum.

\section{Resultados}

Para el análisis de datos se utilizó el programa SIGMASTAT versión 3.5; en todas las pruebas estadísticas el nivel de significancia establecido fue de $p<0.05$.

En primer lugar, se compararon las características demográficas de los grupos constituidos (Tabla 1); por medio de análisis de varianza (ANOVA) de una vía parámetricos y no paramétricos (KruskalWallis), según los requerimientos, se encontró que los grupos de investigación no difirieron significativamente $(p>0.05)$ respecto a edad $\left(F_{[3,76]}\right.$ $=0.918, p=0.436)$, escolaridad $(H=0.470, p$ $=0.925)$, sintomatología depresiva actual determinada por el CES-D $\left(F_{[3,76]}=0.433, p=0.73\right)$, estado cognitivo general evaluado por el EMA $\left(F_{[3,76]}=2.45, p=0.07\right)$ y procesos atencionales valorados por el Test de Cancelación de la "A" (H $=1.295, p=0.730$ ).

\section{Análisis del Desempeño Mnemónico}

El desempeño mnemónico en la prueba Memonum, fue examinado mediante análisis de varianza (ANOVA) de doble vía, de tal manera que el intervalo de exposición de dígitos (1 y 8 segundos) se empleó como Factor 1, el tipo de presentación (presencia y ausencia de distractor) como Factor 2, y los aciertos o los tiempos de respuesta como variable. Cuando ANOVA detectó significancia estadística para el efecto principal de los factores y/o para su interacción, se desarrollaron comparaciones múltiples corregidas empleando la Prueba t de Bonferroni.

Inicialmente, se analizó el número de aciertos, referente a la amplitud de la secuencia numérica evocada correctamente, y el número de aciertos acumulados, definido como la cantidad de números correctamente digitados durante todo el ensayo. 
El ANOVA de doble vía sobre el número de aciertos reveló que existe un efecto significativo del intervalo de exposición $\left(F_{[1,76]}=35.405, p<0.001\right)$, pero no del tipo de presentación $\left(\mathrm{F}_{[1,76]}=3.478, p=\right.$ 0.066). La interacción entre intervalo de exposición y tipo de presentación no resultó estadísticamente significativa $\left(F_{[1,76]}=1.775, p=0.187\right)$. De acuerdo con el análisis post hoc (Prueba $t$ de Bonferroni), el número de aciertos alcanzado por los participantes en el intervalo de 8 segundos fue mayor que el alcanzado por los participantes en el intervalo de 1 segundo $(t=5.95, p<0.001)$ (Figura 1$)$.

En lo referente al número de aciertos acumulados, se detectó (ANOVA de doble vía) un efecto significativo del factor intervalo de exposición $\left(F_{[1,76]}=32.63, p<0.001\right)$ y del factor tipo de presentación $\left(F_{[1,76]}=4.667, p<0.05\right)$. Sin embargo, no se encontraron interacciones significativas entre los factores $\left(F_{[1,76]}=2.087, p=0.153\right)$. La Prueba $t$ de Bonferroni para comparaciones múltiples corregidas, indicó que los participantes obtu- vieron una mayor cantidad de aciertos acumulados en el intervalo de 8 segundos, en comparación con los participantes en el intervalo de 1 segundo $(t=$ $5.712, p<0.001$ ); adicionalmente, el número de aciertos acumulados fue mayor en la presentación sin distractor que en la presentación con distractor $(t=2.16, p<0.05)$ (Figura 2).

Para los tiempos de respuesta, se consideraron las variables: promedio de tiempos de respuesta, tiempo de respuesta mínimo, tiempo de respuesta máximo, y proporción de aumentos y disminuciones de las diferencias de los tiempos de respuesta consecutivos. Las diferencias de los tiempos de respuesta consecutivos fueron procesadas como tiempo de respuesta " $n+1$ ", menos tiempo de respuesta "n". Al analizar mediante ANOVA de doble vía cada una de estas variables, no se evidenció significancia estadística $(p>0.05)$ para el efecto de los factores: intervalo de exposición y tipo de presentación, ni para su interacción.

FIGURA 1

Número de aciertos (Promedio + EEM) según el intervalo de exposición y el tipo de presentación

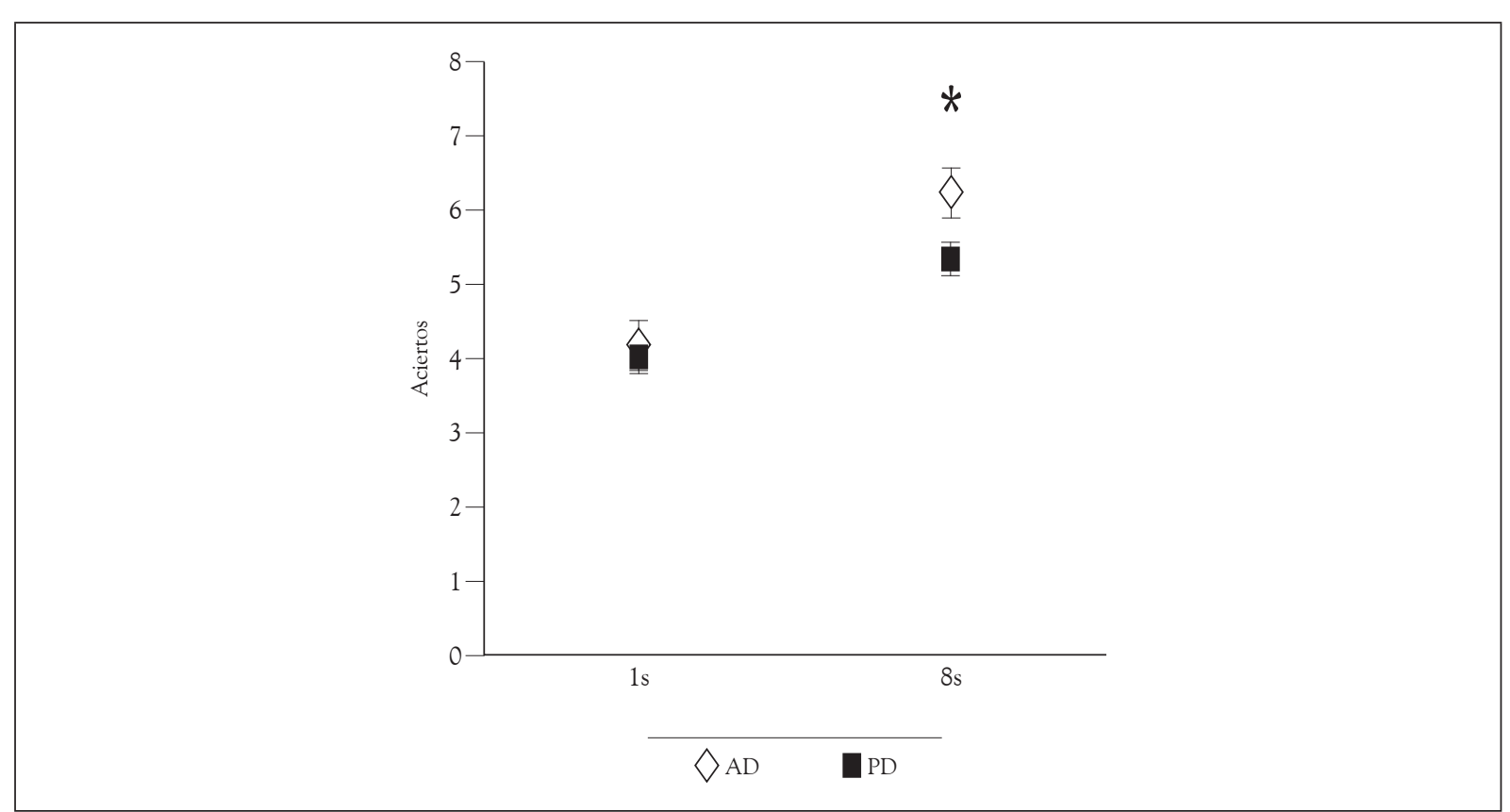

* Mayor número de aciertos en 8 segundos ( $8 \mathrm{~s}$ ) que en 1 segundo (1 s) (ANOVA dos vías, $\mathrm{p}<.001$ ).

Fuente: elaboración propia. 
FIGURA 2

Número de aciertos acumulados (Promedio + EEM) según el intervalo de exposición y el tipo de presentación

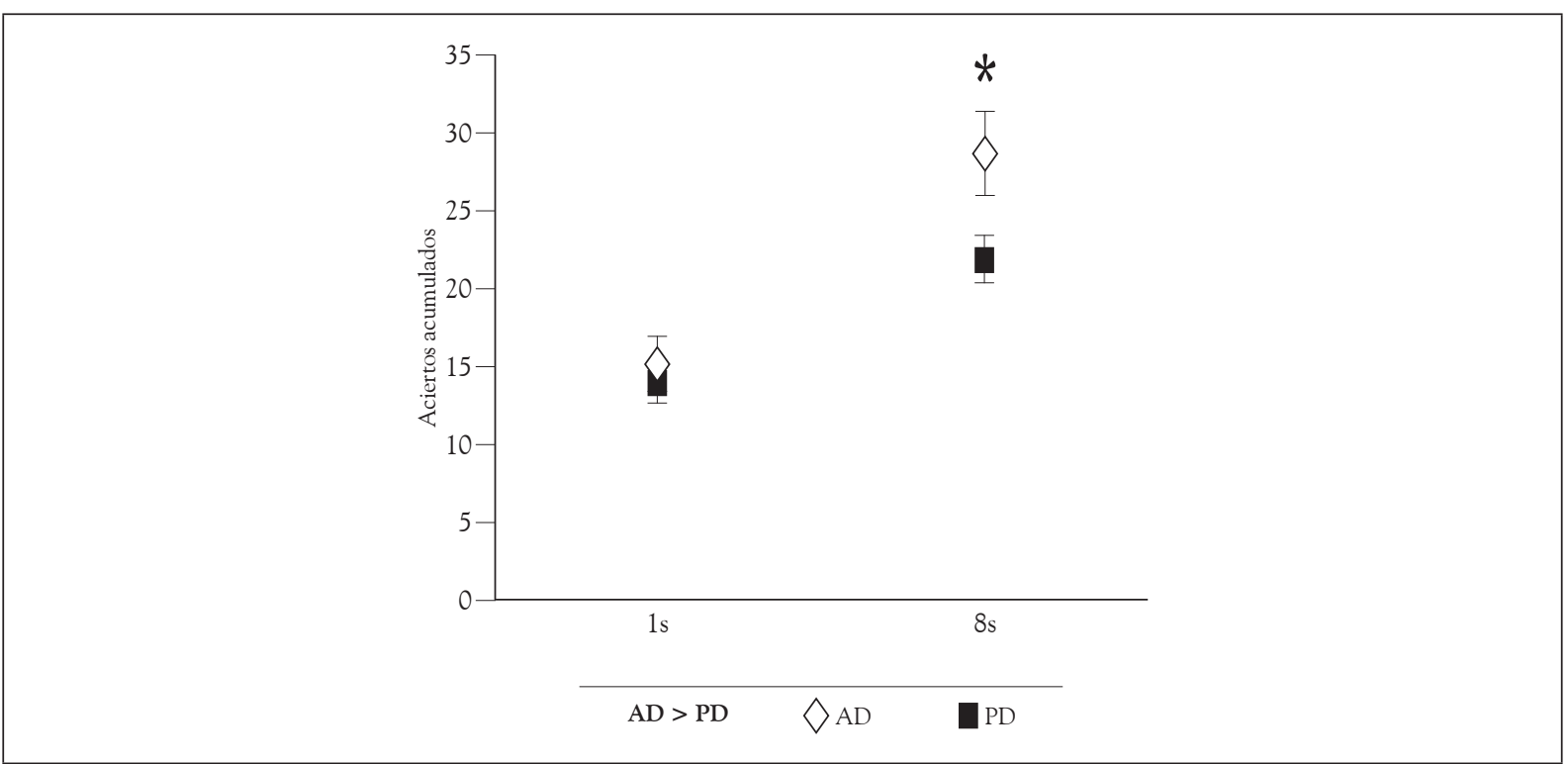

* Mayor número de aciertos acumulados en 8 segundos ( $8 \mathrm{~s}$ ) que en 1 segundo (1 s) (ANOVA dos vías, p <.001).

AD > PD: Mayor número de aciertos acumulados en ausencia de distractor (AD) que en presencia de distractor (PD) (ANOVA dos vías, $\mathrm{p}<0.05$ )

Fuente: elaboración propia.

\section{Análisis de las Variables de la Prueba Memonum}

La prueba ROC fue aplicada sobre las variables de desempeño mnemónico, con el propósito de examinar su capacidad discriminativa respecto al intervalo de exposición ( $1 \mathrm{AD}$ vs. $8 \mathrm{AD} ; 1 \mathrm{PD}$ vs. $8 \mathrm{PD}$ ) y al tipo de presentación (1AD vs. 1PD; $8 \mathrm{AD}$ vs. 8PD). Como se expone en la Tabla 2, los mejores parámetros de discriminación entre intervalos de exposición, con un índice ROC superior a 0.7, fueron los obtenidos por el número de aciertos y el número de aciertos acumulados. En contraste, el análisis de discriminación entre tipos de presentación indicó que ninguna de las variables de desempeño mnemónico presentó un valor superior a 0.7 en el índice ROC.
TABLA 2

Mejores parámetros derivados del análisis ROC para la discriminación entre intervalos

\begin{tabular}{|c|c|c|c|c|c|c|c|}
\hline \multicolumn{8}{|c|}{$\begin{array}{c}\text { Discriminación entre los intervalos de exposición } \\
\text { (1 y } 8 \text { segundos) según el desempeño en la } \\
\text { presentación sin distractor (AD) }\end{array}$} \\
\hline Variable & ROC & $\mathrm{VC}$ & $\begin{array}{c}\text { \% bien } \\
\text { clasificados }\end{array}$ & $\mathrm{S}$ & $\mathrm{E}$ & VPP & VPI \\
\hline ier- & 0.83 & 6.0 & 72.5 & 0.65 & 0.80 & 0.76 & 0.70 \\
\hline $\begin{array}{l}\text { Aciertos } \\
\text { acumu- } \\
\operatorname{lados}^{\text {a }}\end{array}$ & 0.84 & 28.0 & 77. & 0.55 & 1.0 & 1.0 & 0.69 \\
\hline \multicolumn{8}{|c|}{$\begin{array}{l}\text { Discriminación entre los intervalos de exposición } \\
\text { (1 y } 8 \text { segundos) según el desempeño en la } \\
\text { presentación con distractor (PD) }\end{array}$} \\
\hline Tariable & $\mathrm{ROC}$ & VC & $\begin{array}{c}\text { \% bien } \\
\text { clasificados }\end{array}$ & S & $\mathrm{E}$ & VPP & VPN \\
\hline $\begin{array}{l}\text { Cier- } \\
s^{\text {a }}\end{array}$ & 0.82 & 5.0 & 75.0 & 0.85 & 0.65 & 0.71 & 0.81 \\
\hline
\end{tabular}




\begin{tabular}{lllll}
\hline Variable ROC VC $\begin{array}{c}\text { \% bien } \\
\text { clasificados }\end{array}$ & S E VPP VPN \\
\hline
\end{tabular}

Aciertos

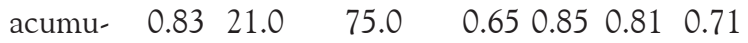

$\operatorname{lados}^{\mathrm{a}}$

Nota ROC = índice ROC; VC = valor de corte; \% bien clasificados = porcentaje de evaluados bien clasificados; $\mathrm{S}$ $=$ sensibilidad $\mathrm{E}=$ especificidad $; \mathrm{VPP}=$ valor predictivo positivo; VPN = valor predictivo negativo.

${ }^{\mathrm{a}} n=40$

Fuente: elaboración propia.

\section{Análisis del Formato de Autoinforme}

Para abordar los datos derivados del Formato de Autoinforme, se realizaron análisis de varianza (ANOVA) de doble vía, designando como Factor 1 el intervalo de exposición de dígitos ( 1 y 8 segundos), como Factor 2 el tipo de presentación (presencia y ausencia de distractor) y como variable los puntajes asignados a las estrategias, distracción o dificultad en la resolución de la Prueba Memonum; seguidos, siempre que fuera necesario, de la prueba $t$ de Bonferroni para comparaciones múltiples corregidas (Figura 3).

\section{Figura 3}

Puntajes asignados a estrategias, nivel de distracción (DST) y nivel de dificultad (DFC) (Promedio \pm EEM), según el intervalo de exposición y el tipo de presentación.

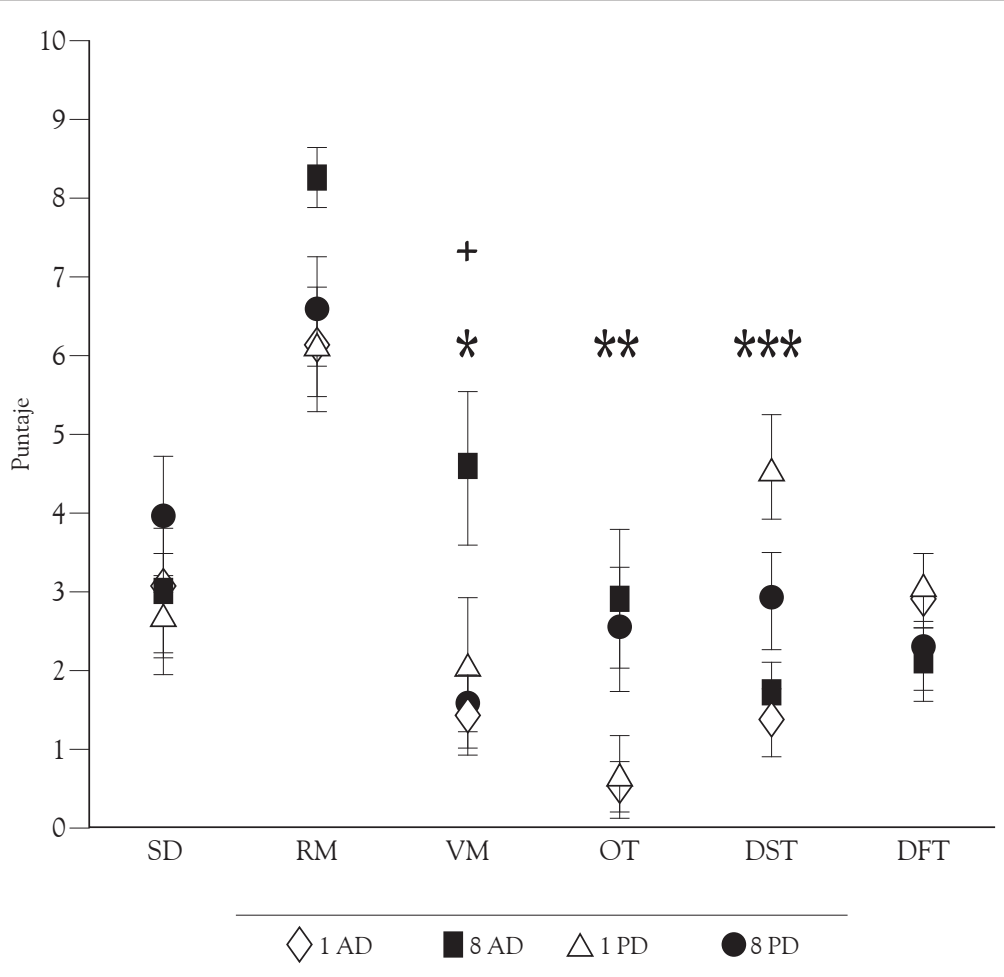

Estrategias: Secuencias de Digitación (SD); Repetición Mental (RM); Visualización Mental (VM); Otras Estrategias (OE).

+ , VM mayor en 8 segundos ausencia de distractor (8AD) que en 8 segundos presencia de distractor (8PD), (ANOVA dos vías, $p<0.05)$.

*, VM mayor en 8 segundos ausencia de distractor (8AD) que en 1 segundo ausencia de distractor (1AD), (ANOVA dos vías, $p$ $<0.05)$.

**, OT mayor en 8 segundos (8AD-8PD) que en 1 segundo (1AD-1PD), (ANOVA dos vías, $p<0.05)$.

***, DST mayor en presencia de distractor (1PD-8PD) que en ausencia de distractor (1AD-8AD) (ANOVA dos vías, $p<0.001$ ).

Fuente: elaboración propia. 
El ANOVA de doble vía sobre cada una de las estrategias, no reveló diferencias estadísticamente significativas $(p>.05)$ para Repetición Mental y Secuencias de Digitación, atribuibles a los factores intervalo de exposición y tipo de presentación, ni a su interacción.

Sin embargo, para la estrategia Visualización Mental detectó interacciones significativas entre el intervalo de exposición y el tipo de presentación $\left(F_{[1,76]}=5.884, p<0.05\right)$, aunque no evidenció efectos principales del factor intervalo de exposición, ni del factor tipo de presentación $\left(F_{[1,76]}\right.$ $=3.102, p=0.082 ; F_{[1,76]}=2.439, p=0.122$, respectivamente). Las comparaciones múltiples corregidas (Prueba $t$ de Bonferroni) indicaron que al interior del grupo de participantes que ejecutaron la prueba Memonum en el intervalo de 8 segundos, los evaluados en la presentación sin distractor (8AD) atribuyeron una mayor puntación a la estrategia Visualización Mental, comparados con los evaluados en la presentación con distractor (8PD) $(t=2.82, p<0.05)$; así mismo, al interior del grupo de participantes que desarrollaron la prueba Memonum en ausencia de distractor, los evaluados asignaron una mayor puntación a la estrategia Visualización Mental en el intervalo de 8 segundos ( $8 \mathrm{AD}$ ) en comparación con los evaluados en el intervalo de 1 segundo (1AD) $(t=$ 2.961, $p<0.05$ ).

Respecto a la variable Otras Estrategias, este mismo tipo de análisis mostró un efecto estadísticamente significativo del intervalo de exposición $\left(F_{[1,76]}=10.286, p<0.05\right)$, pero no del tipo de presentación $\left(F_{[1,76]}=0.0223, p=0.882\right)$. Por su parte, la interacción entre intervalo de exposición y tipo de presentación no resultó significativa $\left(F_{[1,76]}\right.$ $=0.2, p=0.656)$. La prueba $t$ de Bonferroni para comparaciones múltiples corregidas, reveló que los participantes atribuyeron mayor puntaje al uso de otras estrategias en el intervalo de 8 segundos, que los participantes en el intervalo de 1 segundo $(t=$ 3.207, $p<0.05$ ).

Por otro lado, al analizar el nivel de distracción (ANOVA de doble vía), se encontró que el factor tipo de presentación tuvo un efecto significativo $\left(F_{[1,76]}=16.147, p<0.001\right)$; sin embargo, ni el factor intervalo de exposición $\left(F_{[1,76]}=1.635, p\right.$ $=0.205)$, ni la interacción entre factores $\left(F_{[1,76]}\right.$ $=3.336, p=0.072)$ mostraron significancia estadística. Según el análisis post hoc (Prueba $t$ de Bonferroni), los participantes que presentaron la prueba Memonum con distractor, asignaron un mayor puntaje al nivel de distracción, en comparación con los participantes que ejecutaron la prueba $\sin$ distractor $(t=4.018, p<0.001)$.

Finalmente, en relación al nivel de dificultad, el ANOVA de doble vía no detectó efectos principales de los factores intervalo de exposición y tipo de presentación, ni interacciones entre ellos $(p>0.05)$.

\section{Análisis de Correlación}

Fue empleado el coeficiente Pearson con la finalidad de estimar la relación de variables del desempeño en la prueba Memonum (número de aciertos, número de aciertos acumulados, tiempo de respuesta mínimo, promedio de los tiempos de respuesta) con las medidas de sintomatología depresiva actual (puntaje en CES-D), estado cognoscitivo global (puntaje en EMA) y procesos atencionales (puntaje en sección de atención y cálculo del EMA -EMA.AC-, puntaje y tiempo de ejecución en el Test de Cancelación de la "A" -TCA y TCA.TE, respectivamente-).

Los análisis efectuados revelaron que las variables de desempeño mnemónico de la Prueba Memonum no correlacionaron de manera significativa con CES-D ( $p>0.05)$.

Sin embargo, el número de aciertos y el número de aciertos acumulados presentaron una correlación positiva estadísticamente significativa con EMA $(r=0.428, p<0.01 ; r=0.428, p<0.01)$, EMA.AC $(r=0.392, p<0.01 ; r=0.384, p<$ $0.01)$ y TCA $(r=0.296, p<0.01 ; r=0.257, p$ $<0.05)$.

Adicionalmente, el tiempo de respuesta mínimo y el promedio de tiempos de respuesta mostraron una correlación negativa estadísticamente significativa con EMA $(r=-0.389, p<0.01 ; r=$ $-0.365, p<.01)$, EMA.AC $(r=-0.314, p<0.01 ; r$ $=-0.334, p<0.01)$, y TCA $(r=-0.655, p<0.01$; 
$r=-0.564, p<0.01)$, y una correlación positiva estadísticamente significativa con TCA.TE $(r=$ $0.305, p<0.01 ; r=0.223, p<0.05)$.

De acuerdo con los anteriores resultados, a mayor puntaje obtenido en las medidas para la valoración del estado cognitivo global (EMA) y de procesos atencionales (EMA.AC y TCA), mayor capacidad de almacenamiento de dígitos y menores tiempos de respuesta en la Prueba Memonum; así mismo, el incremento del tiempo de ejecución en el Test de Cancelación de la "A" se asocia con un incremento de los tiempos de respuesta en la Prueba computarizada.

\section{Análisis de Regresión Lineal Múltiple}

Se implementó el método de regresión lineal múltiple, designando como variable de salida el número de aciertos alcanzado por los participantes en la Prueba Memonum, y como variables predictoras: la edad, la escolaridad, el intervalo de exposición de dígitos, el tipo de presentación en la prueba Memonum, el puntaje en CES-D, el puntaje total en EMA, el puntaje de las distintas secciones del EMA, el puntaje y tiempo de ejecución del Test de Cancelación de la "A".

Los resultados derivados de dicho análisis, evidenciaron significancia estadística $\left(F_{[3,76]}=19.91\right.$, $p<0.001)$ para el modelo que incluye el intervalo de exposición, el puntaje en el Test de Cancelación de la "A" (TCA), y la sección de atención y cálculo del EMA (EMA.AC); variables que de forma conjunta explican el $42 \%$ de la varianza del número de aciertos en la Prueba Memonum $\left(R^{2}\right.$ ajustado $=0.418)$; alcanzando una predicción de la variable de salida que no logra ser mejorada de forma significativa al relacionar las restantes variables predictoras. La ecuación correspondiente al modelo ajustado fue la siguiente:

Número de aciertos $=-0.518+(0.217 *$ Intervalo $)+(0.243 *$ TCA $)+(0.243 *$ EMA.AC $)$.

De forma complementaria, se realizó un análisis de regresión lineal múltiple, utilizando las mismas variables predictoras previamente señaladas, y definiendo como variable de salida el tiempo de respuesta mínimo en la Prueba Memonum.
Los resultados indicaron que el modelo constituido por el puntaje en el Test de Cancelación de la "A" (TCA), y la sección de atención y cálculo del EMA (EMA.AC) fue estadísticamente significativo $\left(F_{[2,77]}=32.970, p<0.001\right)$; explicando un $45 \%$ de la varianza del tiempo de respuesta mínimo $\left(R^{2}\right.$ ajustado $\left.=.447\right)$; sin presentarse ajustes significativos en dicho modelo al relacionar las restantes variables predictoras. La ecuación obtenida fue:

Tiempo de respuesta mínimo $=2.859-(0.145$ * TCA $)-(0.0421 *$ EMA.AC).

\section{Discusión}

De acuerdo con el objetivo de este estudio, merece señalarse que las diferencias observadas en el desempeño mnemónico de la Prueba Memonum son atribuibles al efecto del intervalo de exposición de dígitos, al tipo de presentación de la prueba computarizada y/o a su interacción; puesto que los grupos de participantes no difirieron significativamente respecto a edad, escolaridad, sintomatología depresiva actual, estado cognoscitivo global y procesos atencionales, sugiriendo que estas características no actuaron como variables extrañas.

La presente investigación reveló un mejor desempeño mnemónico en mujeres mayores de 50 años, presentando la prueba Memonum en el intervalo de exposición de 8 segundos, reflejado en una mayor cantidad de aciertos y un mayor número de aciertos acumulados, en comparación con las participantes asignadas al intervalo de 1 segundo.

Este hallazgo soporta la propuesta de que en amplios tiempos de exposición mejora el desempeño mnemónico potenciando la capacidad de almacenamiento, confirmada por Fischer (2001) quien empleó una versión computarizada de la tarea visoespacial de Cubos de Corsi en tres intervalos de exposición (1, 3 y 9 segundos), y corroborada por el estudio de Albarracín et al. (2008), utilizando la prueba Memonum con intervalos de exposición de dígitos de 1,8 y 16 segundos, en una muestra de jóvenes universitarios.

Autores como Emery et al. (2007) exponiendo secuencias dígito-letra en tres intervalos (750, 
1750 y 2750 milisegundos), demostraron un incremento en la capacidad de almacenamiento de adultos jóvenes y adultos mayores, si solicitaban como respuesta la manipulación de ítems en orden progresivo (dígitos) y en orden alfabético (letras). Así se considera que la potenciación mnemónica asociada a intervalos amplios involucra el empleo de estrategias mnemónicas.

Salthouse (1996) plantea que este incremento se debe a un procesamiento de información más elaborado; Repovs y Baddeley (2006) afirman que corresponde con la característica de la memoria de trabajo para procesar y almacenar información en paralelo. El aumento en el tiempo de exposición facilita el contacto con el estímulo, momento durante el cual, además de percibirse la información, estaría disponible lo suficiente para realizar simultáneamente operaciones cognitivas como asociaciones o manipulaciones estratégicas (Salthouse, 1996).

Lo recién postulado se refleja en el reporte de estrategias para desarrollar la prueba Memonum, pues se evidenció que los participantes expuestos al intervalo de 8 segundos atribuyeron una puntuación significativamente superior en el uso de estrategias adicionales, comparada con las puntuaciones de los evaluados en el intervalo de 1 segundo.

De hecho, el empleo de estrategias mnemónicas asociadas a la manipulación del tiempo de exposición a estímulos, son condiciones estimadas en programas computarizados de entrenamiento cognitivo para adultos mayores (Günther et al., 2003), o en intervenciones de rehabilitación cognitiva asistida por computadora (Lynch, 2002).

Los resultados ofrecidos junto con la evidencia de un mejoramiento mnemónico en función de mayores intervalos de contacto con la información, posibilitan la propuesta del tiempo de exposición como recurso potenciador de la capacidad de almacenamiento en la memoria de trabajo de adultos mayores; relativizando la postura de una limitación definitiva de la capacidad mnemónica durante un envejecimiento normal (Román \& Sánchez, 1998).

Respecto al análisis de variables relacionadas con tiempos de respuesta como: el promedio de latencia de digitación, el tiempo de respuesta máximo y mínimo, la proporción de disminuciones y la proporción de aumentos de tiempos de respuesta, no se evidenciaron diferencias significativas como efecto de la exposición a los intervalos de 1 y 8 segundos. Resultados similares ofreció Albarracín et al. (2008) implementando la prueba Memonum en jóvenes universitarios, pues no hallaron diferencias significativas entre la exposición al intervalo de 1 y 8 segundos con las variables antes mencionadas.

La implementación de la prueba Memonum en adultos jóvenes sugirió que un intervalo de exposición de 8 segundos, resulta insuficiente para potenciar un desempeño mnemónico reflejado en latencias de digitación significativamente menores; en consecuencia, se plantea el empleo de mayores tiempos de exposición, para apreciar un efecto del intervalo sobre variables relacionadas con tiempos de respuesta, pues Albarracín et al. (2008) reveló diferencias significativas, comparando las aceleraciones entre intervalos de 1 y 16 segundos, siendo mayores para este último tiempo de exposición.

Según Fischer (2001) se esperaría que una tarea bajo la condición de amplios intervalos de exposición se desarrollara con menores tiempos de respuesta; no obstante, su estudio con tres intervalos de exposición (1, 3 y 9 segundos) utilizando la versión computarizada del test de los Cubos de Corsi en estudiantes universitarios, no mostró diferencias significativas, tomando como medida, el tiempo de respuesta inicial.

Asimismo, Fischer (2001) afirma que la variable de tiempo de respuesta que empleó, resultó menos sensible que la clásica cantidad de ítems evocados para apreciar los efectos de los intervalos de exposición; apoyando así medidas del test Memonum relacionadas con la capacidad de almacenamiento como el número de aciertos y el número de aciertos acumulados, pues el análisis ROC indicó que estas variables se constituyen en parámetros efectivos para discriminar si el rendimiento obtenido por un participante corresponde a un intervalo de 1 u 8 segundos.

En cuanto a los resultados sobre el tipo de presentación, los participantes mostraron un mejor desempeño mnemónico en ausencia del distrac- 
tor, evidenciado en un mayor número de aciertos acumulados en contraste con las cantidades alcanzadas por los evaluados en la presentación con distractor en la prueba Memonum.

El hallazgo de esta diferencia en la capacidad de almacenamiento como efecto del distractor, no se determinó en el estudio de Albarracín et al. (2008) empleando la prueba Memonum en jóvenes universitarios, argumentando que la habilidad de la memoria de trabajo reside en mantener activa temporalmente una información, incluso ante la presencia de un distractor (Engle et al., 1999 citados en Albarracín et al., 2008).

En este sentido, la presencia de distractor repercutió en una menor cantidad de aciertos acumulados obtenida por los participantes, un mayor puntaje asignado a la distracción durante la prueba y una menor utilización de estrategias visuales para la memorización. Esto sugiere la afectación del mecanismo atencional como explicación de los cambios de la memoria asociados a una edad madura, característica de la muestra evaluada. Entre los cambios neuropsicológicos durante un envejecimiento normal, Román y Sánchez (1998), señalan variaciones morfológicas cerebrales en la corteza frontal y prefrontal, relacionadas con funciones cognitivas como la planificación, el autocontrol, la organización y procesos de inhibición; desde autores como Repovs y Baddeley (2006) y Gathercole (1994) este sustrato neurológico estaría comprometido a nivel de funcionamiento y representación con el componente ejecutivo central.

Así, según Pousada (1998) un déficit en mecanismos atencionales ejercidos por el sistema ejecutivo, influye en el control inhibitorio, incrementa los contenidos en la memoria de trabajo incorporando información no relevante, provocando mayor competición en la recuperación de información relevante, reflejándose en olvidos habituales, frecuentes respuestas inapropiadas, o disminución en el volumen de almacenamiento.

Lo antepuesto es coherente con el puntaje asignado en el autoinforme, pues los evaluados percibieron un mayor nivel de distracción en el tipo de presentación con presencia de distractor, en contraste con participantes que presentaron la prueba Memonum en ausencia del distractor. Según lo postulan Pousada (1998) y Rodríguez et al. (2005), las presentaciones que emplean distractores comprometen notablemente procesos atencionales, tal y como lo demuestra la prueba Memonum, considerando su utilidad para la evaluación de déficits de atención.

Sin embargo, el efecto del distractor sobre el desempeño mnemónico ha diferido en estudios con la prueba Memonum. En jóvenes universitarios, afectó variables relacionadas con tiempos de respuesta (Albarracín et al., 2008), reflejando el requerimiento de mayor tiempo para digitar las series numéricas, indicando un procesamiento dirigido a mantener la atención en la serie de dígitos ante los cambios de color en la pantalla. Por el contrario, en la presente investigación con mujeres mayores de 50 años, el distractor tuvo un efecto sobre la capacidad de almacenamiento, aunque sin afectar significativamente variables relacionadas con tiempos de respuesta.

Una posible explicación se deriva de los procesos involucrados en los tiempos de respuesta. Según Sainz (1992), mayores tiempos de respuesta son consecuencia de un procesamiento de la memoria de trabajo que implica funciones atencionales como la inhibición de distractores, y a su vez el mantenimiento activo de la información relevante. Siendo así, no hallar mayores tiempos de respuesta asociados a la presentación con distractor en este estudio, refleja un procesamiento realizado de forma insuficiente para inhibirlo, afectando la capacidad de almacenamiento evidenciada en un menor número de aciertos acumulados; atribuyendo este resultado a un déficit en mecanismos de control atencional asociados al envejecimiento (Pousada, 1998).

Estos postulados perfilan la intervención esencial de procesos atencionales sobre el desarrollo de tareas mnemónicas (Estévez et al., 1997), confirmado desde correlaciones significativamente positivas entre medidas atencionales como el test de cancelación de la "A" y la sección de atención y cálculo del EMA, con el número de aciertos en la prueba Memonum; y significativamente negativas, con variables de tiempos de respuesta como 
el promedio de digitación y la mínima latencia. Más aún, con base en los modelos de regresión lineal realizados, se considera el rendimiento en los mencionados test atencionales como las medidas que mejor explican la varianza del desempeño en variables de almacenamiento como el número de aciertos y de tiempos de respuesta, como el tiempo de respuesta mínimo en la prueba Memonum.

Las anteriores evidencias conducen a estimar que el desempeño en los test atencionales utilizados, se convierte en medida predictora del desempeño en la prueba Memonum, relación razonable teniendo en cuenta que la tarea cancelación de la "A", evalúa atención visual sostenida, exigiendo la localización de una letra particular, y selectiva, requiriendo a su vez la inhibición de estímulos irrelevantes (Ardila et al., 1994; Lezak, 1995); y la sección de atención y cálculo del EMA, análoga al test de sustracción seriada implica la retención por corto plazo de cantidades numéricas, examinando además el volumen atencional (Estévez et al., 1997).

El soporte ofrecido junto con los resultados, conllevan a dos reflexiones sobre la memoria de trabajo. La hipótesis de cambios en la memoria en un envejecimiento normal asociados a fallas en el componente ejecutivo, afectando operaciones cognitivas como: la planeación estratégica, la organización de la información y los procesos inhibitorios (Román \& Sánchez, 1998). Y el reconocimiento de una intervención atencional relevante sobre el desempeño mnemónico en la tarea Memonum, conduciendo al planteamiento de una función atencional análoga al papel del ejecutivo central (Benítez \& Bajo, 1998; Gathercole, 1994).

Finalmente, los hallazgos de esta investigación perfilan a la prueba Memonum como herramienta de evaluación cognitiva, estimando el efecto de un mayor intervalo de exposición sobre procesos de la memoria de trabajo en adultos mayores, y además permiten proponer su aplicación en el estudio, evaluación y diagnostico de déficits atencionales, considerando los efectos de la modalidad de presentación con distractor, sobre el desempeño mnemónico.

\section{Referencias}

Albarracín, A., Dallos, M. \& Conde, C. (2008). Implementación de una prueba automatizada para la evaluación de memoria operacional: Memonum. Revista Colombiana de Psiquiatría, 37, 169-181.

Ardila, A. \& Rosselli, M. (1994). Neuropsicología Clínica. Medellín, Colombia: Prensa Creativa.

Ardila, A., Rosselli, M. \& Puente, A. (1994). Neuropsychological evaluation of the spanish speaker. New York: Plenum Press.

Baddeley, A. (1999). Memoria humana. Teoría y práctica. Madrid, España: McGraw Hill.

Benítez, P. \& Bajo, T. Hacia un modelo de memoria y atención en interpretación simultánea. Quaderns, 2, 107-117.

Burin, D. \& Duarte, A. (2005). Efectos del envejecimiento en el ejecutivo central. Revista Argentina de Neuropsicología, 6, 1-11.

Crook, T., Barthus, R. T., Ferris, S. H., Whitehouse, P., Cohen G. D. \& Gershon, S. (1986). Age Associated Memory Impairment: Proposed diagnostic criteria measures of clinical change. Report of a Nacional Institute of Mental Health work group. Developmental Neuropsychology, 2, 261-276.

Emery, L., Myerson, J. \& Hale, S. (2007). Age differences in item manipulation span: The case of letter-number sequencing. Psychology and Aging, 22, 75-83.

Estévez, A., García, C. \& Junqué, C. (1997). La atención: una compleja función cerebral. Revista de Neurología, 25 (148), 1989-1997.

Etchepareborda, M. \& Abad-Mas, L. (2005). Memoria de trabajo en los procesos básicos del aprendizaje. Revista de Neurología, 40, 79-83.

Fischer, M. (2001). Probing spatial working memory with the Corsi Blocks Task. Brain and Cognition, 45, 143-154.

Folstein, M., Folstein, S. \& McHugh, P. (1975). "MiniMental State". A practical method for grading the cognitive state of patients for the clinician. Journal Psychiatric Research, 12, 189-198.

Gathercole, S. (1994). Neuropsychology and working memory: A review. Neuropsychology, 8, 494-505.

Gazzaley, A., Sheridan M., Cooney J. \& D'Esposito M. (2007). Age-related deficit in component pro- 
cesses of working memory. Neuropsychology, 21 (5), 532-539.

Günther, V., Shafer, P., Holzner, B. \& Kemmler, G. (2003). Long-term improvements in cognitive performance through computer-assisted cognitive training: A pilot study in a residential home for older people. Aging E Mental Health, 7, 200-206.

Lezak, M. (1995). Neuropsychologycal assesment (3a. ed.). New York: Oxford University Press.

Lynch, B. (2002). Historical Review of Computer - assisted Cognitive Retraining. Journal Head Trauma Rehabilitation, 17, 446-457.

Orozco, M., Orozco, L., Herrera, J., Méndez, G., Mendoza, M., Pinzón, L. et al. (2004). Validación de un instrumento para medir la calidad de vida en sus aspectos físico, emocional, cognitivo y social en personas ancianas. Revista Asociación Colombiana de Gerontología y Geriatría, 18 (1-2), 606-612.

Pousada, M. (1998). El Déficit en los mecanismos de inhibición como hipótesis explicativa de la pérdida de memoria asociada a la edad. Anales de Psicología, 14, 55-74.

Pradilla, G., Vesga, B., León-Sarmiento, F., Bautista, L., Núñez, L., Vesga, E. et al. (2002). Neuroepidemiología en el oriente colombiano. Revista de Neurología, 34, 1035-1043.

Radloff, L. (1977). The CES-D scale: A self-report depression scale for research in the general po- pulation. Applied Psychological Measurement, 1, 385-401.

Repovs, G. \& Baddeley, A. (2006). The multi-component model of working memory. Neuroscience, 139, 5-21.

Rodríguez, J., Fajardo, G. \& Mata, P. (2006) Sistema automatizado para el estudio de la memoria visual a corto plazo. Revista Artemisa, 7, 108-117.

Román, F. \& Sánchez P. (1998). Cambios neuropsicológicos asociados al envejecimiento normal. Anales de la Psicología, 14, 27-43.

Roselli, D., Ardila, A., Pradilla, G., Morillo, L., Bautista, L., Rey, O. et al. (2000). El examen mental abreviado (Mini-Mental State Examination) como prueba de selección para el diagnóstico de demencia: estudio poblacional colombiano. Revista de Neurología, 30, 428-432.

Sainz, C. (1992). La velocidad de anticipación en alumnos de EGB. Tesis doctoral, Universidad Complutense de Madrid, España. Recuperado el 9 de mayo, 2008, de http://eprints.ucm.es/ tesis/19911996/S/5/S5002001.pdf

Salthouse, T. (1996). The processing-speed theory of adult age differences in cognition. Psychological Review, 103 (3), 403-428.

Uribe, A., Valderrama, L. \& Molina J. (2007). Salud objetiva y salud psíquica en adultos mayores colombianos. Acta Colombiana de Psicología, 10, 75-81. 